\title{
Biogenesis of Mitochondria in Trichoderma viride: Structural Changes in Mitochondria and Other Spore Constituents during Conidium Maturation and Germination
}

\author{
By DAlia Rosen, M. EDELMAN, E. GALUN and D. DANON \\ Departments of Plant Genetics and Biological Ultrastructure, \\ Weizmann Institute of Science, Rehorot, Israel
}

(Receired 28 August 1973)

\begin{abstract}
SUMMAR Y
Structural changes in mitochondria and other spore constituents during maturation and germination of Trichoderma viride conidia were investigated. During maturation there was an increase in spore volume and number of mitochondria, while endoplasmic reticulum and electron-dense bodies disappeared. The stellate, precursor-lipid-granules underwent structural and chemical changes, the smooth plasmalemma invaginated and the conidial wall became thicker and relatively impermeable. The resting conidium contained few internal structures - only a single nucleus, several ovoid mitochondria and a few lipid granules.

After inoculation in germination medium there was a $4 \mathrm{~h}$ lag period, during which the conidia did not increase significantly in size, take up cotton blue or undergo a change in mitochondrial number. However, metabolic activity was evident from the reappearance of the electron-dense bodies, a change in the shape of some of the mitochondria and an increased permeability to the various fixation and embedding reagents.

The period of conidium swelling was accompanied by a reappearance of the endoplasmic reticulum, often in association with and seemingly interconnecting the various organelles. The lipid granules gradually disappeared and the electrondense bodies became partially evacuated. Mitochondria increased in number, many having cup-shaped configuration; an unbalanced growth of surface membranes with respect to matrix may have been the physical cause for this shape. The nucleus divided at least once before the emergence of the germ tube. Structural changes continued during germ-tube emergence. The mother conidium in Trichoderma retained its ultrastructural integrity, and did not vacuolate.
\end{abstract}

INTRODUCTION

The biogenesis of mitochondria has been actively investigated in recent years, attention being focused on fungal systems (for reviews see Küntzel, I97 I Linnane, Haslam, Lukins \& Nagley, 1972). Germinating mould conidia afford a simple example of differentiation. Germination is accompanied by a vast increase in aerobic respiration and oxygen consumption (Cochrane, 1966; Gottlieb, 1966), and by a burst of activity of mitochondrial enzymes (Weiss, I963; Ohmori \& Gottlieb, 1965). This indicates the involvement of mitochondria in the process of conidium differentiation from the resting stage to a metabolically active vegetative state. Several aspects of this transition have been investigated: synthesis of macromolecules and changes in enzyme activity (Gottlieb, I966), environmental requirements (Martin \& Nicolás, 1970) and structural changes (Hawker, 1966; Gull \& Trinci, 1971). However, very little information is available on the structural changes and biochemical activity of the mitochondria during this process. 
Trichoderma viride has several features which make it a promising organism for studying mitochondrial biogenesis and its involvement in fungal germination. It has uninucleated conidia, vast crops of which can be obtained by a simple photoinduction (Gressel \& Galun, 1967; Weinman-Greenshpan \& Galun, 1969) so that conidial masses can be collected at the required stages of maturation. Methods for the study of mitochondrial nucleic acids in Trichoderma have been worked out (Edelman, Verma, Herzog, Galun \& Littauer, 1971), and the processing of nucleic acids during conidium germination has recently been investigated in our laboratory (S. Spiegel and R. Stavy, unpublished).

As a prologue to the study of several aspects of mitochondrial biogenesis, this work concentrated on the structural changes occurring during conidium development, starting with the immature conidium and ending just after protrusion of the germ tube. The structural transitions of the mitochondria were investigated with reference to the concomitant structural changes in the other constituents of the differentiating conidium.

\section{METHODS}

Stock cultures. Trichoderma viride Pers. ex Fries (fungi imperfecti) isolate No. 2042 was used in all experiments. Stock cultures were obtained by inoculating $100 \mathrm{~mm}$ Petri dishes containing $10 \mathrm{ml}$ potato dextrose agar (Difco) with conidia and incubating at $24 \pm \mathrm{I}$ C for about 2 days. Vegetative colonies of about $5 \mathrm{~cm}$ diameter were produced and stored at 2 to $4{ }^{\circ} \mathrm{C}$ until required.

Production of conidia. Agar blocks (about $2 \mathrm{~mm}^{3}$ ) were removed from the periphery of the above cultures and placed at the centre of $100 \mathrm{~mm}$ Petri dishes containing $10 \mathrm{ml}$ potato dextrose agar enriched with $0.5 \%$ Difco Bacto peptone and $0.5 \%$ Difco yeast extract, and incubated at $24 \pm \mathrm{I}{ }^{\circ} \mathrm{C}$ in the dark. After 40 to $45 \mathrm{~h}$ the colonies were exposed to fluorescent light for $2 \mathrm{~min}$ and returned to darkness. This treatment induces a ring of conidia which are of about the same age (Galun \& Gressel, 1966; Gressel \& Galun, 1967; Kumagai \& Oda, r969). Immature conidia (white) or mature conidia (green) were harvested at various times after photoinduction by gently agitating colonies with sterile, distilled water. The suspension was strained through four layers of Batiste to remove mycelial fragments. The conidia were then used immediately.

Germination. Conidia were washed three times by centrifugation and resuspension in distilled water, and inoculated into $500 \mathrm{ml}$ Erlenmeyer flasks containing $50 \mathrm{ml}$ potato dextrose broth (Difco) enriched with $0.5 \%$ peptone and $0.5 \%$ yeast extract (germination medium), to give $\mathrm{I} \times 10^{7}$ conidia $/ \mathrm{ml}$. The flasks were incubated at $25 \pm \mathrm{I}{ }^{\circ} \mathrm{C}$ on a rotary shaker at I $40 \mathrm{rev} . / \mathrm{min}$ and samples were removed at intervals.

Determination of swelling and germ-tube emergence. At various times after inoculation in germination medium one drop of conidial culture was suspended in $0.5 \mathrm{ml}$ of a $20 \%$ solution of cotton blue in lactophenol. After $5 \mathrm{~min}$ at room temperature the stained suspension was diluted I:20 with distilled water, centrifuged and the pellet washed with distilled water. Swollen conidia absorbed the dye while resting conidia did not (Ekundayo \& Carlile, 1964). Four hundred conidia were counted in each sample by means of a haemocytometer. If the counting had to be postponed for a few hours, the stained spores were kept over ice.

Electron microscopy. Immature conidia and conidia incubated for different periods in germination medium were fixed for $2 \mathrm{~h}$ at room temperature in $2.5 \%$ glutaraldehyde in $0 \cdot$ I M-cacodylate buffer, $\mathrm{pH} 7 \cdot 2$. This was followed by an overnight wash in cacodylate buffer (five changes), postfixation in $\mathrm{I} \% \mathrm{KMnO}_{4}$ and $0.9 \% \mathrm{NaCl}$ for $30 \mathrm{~min}$, washing with the above buffer, and then a further postfixation in $2 \% \mathrm{OsO}_{4}$ in water for $\mathrm{I}_{5} \mathrm{~min}$ followed by 
washing in distilled water for $5 \mathrm{~min}$. After dehydration in a graded ethanol series, terminated by two changes of propylene oxide, conidia were infiltrated with and embedded in Araldite (Fluka-Europan ACM). Polymerization was carried out at 40 and 50 C for $\mathrm{I}$ day each and at $60 \mathrm{C}$ for 5 days. Permanganate was used in the fixation procedure in order to obtain well-contrasted membranous structures.

The mature, resting conidia presented special problems because of their extreme impermeability to the above fixatives and embedding solutions. Fixation was achieved by mechanically cracking the spore wall at $4000 \mathrm{rev} . / \mathrm{min}$ for $3 \mathrm{~min}$ with cooling, in a Braun Homogenizer (Model MSK). For this, conidia were suspended in $5 \mathrm{ml}$ of fixative and introduced into $30 \mathrm{ml}$ homogenizer bottles together with $350 \mathrm{mg}$ glass beads $(0.25$ to $0.30 \mathrm{~mm}$ diam). The fixation then proceeded as described above.

Sections were cut with a glass knife, on either a Danon Ultramicrotome or a LKB Ultratome, mounted upon colodion-coated copper grids, stained either with saturated uranyl acetate solution in $50 \%$ ethanol followed by alkaline lead citrate (Reynolds, 1963) or with lead citrate alone, and examined in a JEM-T7 electron microscope at $60000 \mathrm{~V}$.

Measurements. The diameters of about 30 conidia at each developmental stage were measured from electron micrographs of median or near-median sections and the average values calculated. The volumes of the conidia were calculated by assuming they have an ellipsoidal shape. The volumetric densities of the various components within the conidia and the mitochondrial surface density were calculated by means of Weibel's stereological method (Weibel, Kistler \& Scherle, 1966). For volumetric density measurements a regular point lattice was randomly superimposed on random micrographs of random sections and the points lying on transections of the structures of interest were counted. For surface density measurements a test line was randomly placed on random sections and the number of intersections between the test line and the contour of the mitochondrial transections was counted.

Three-dimensional reconstruction. Slabs of FIMO modelling material (EFA Eberhard Faber, Germany) were cut into the shapes of mitochondrial figures appearing in electron micrographs of serial sections. The slab thickness was such that the bodies obtained emulated the corresponding slices three-dimensionally to scale. Serial slice models were then assembled to obtain approximate reconstructions to scale of entire mitochondria.

\section{RESULTS \\ Kinetics of conidial germination}

Figure 1 shows the germination course of a typical population of Trichoderma viride conidia. After a lag period lasting about $4 \mathrm{~h}$ the resting conidia noticeably start to swell. The swelling process was followed by the uptake of cotton blue (Ekundayo \& Carlile, 1964) as well as by measurements from electron micrographs. Although increase in conidial volume started $4 \mathrm{~h}$ after incubation in germination medium, ultrastructural and biochemical changes could be detected as little as $2 \mathrm{~h}$ after inoculation (see below). By $8 \mathrm{~h}$ virtually all the conidia had absorbed the dye and the average volume was 3.8 times that of the resting conidia (see Table 2 below). The swelling of fungal spores has been shown by many investigators to be more than merely the physical absorption of water; rather, it is a very active metabolic process requiring energy (Cochrane, 1966; Gottlieb, 1966) and occurring concomitantly with ultrastructural changes (e.g. Hawker, Thomas \& Beckett, 1970; Fletcher, 1971; Gull \& Trinci, 1971; McCoy, Girard \& Kornfeld, 1971). 


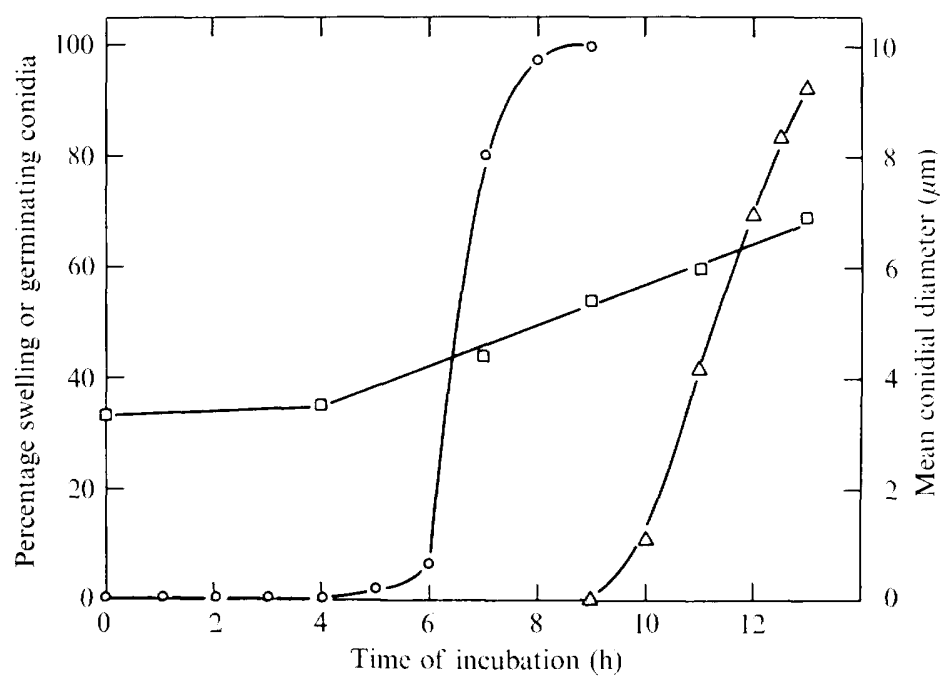

Fig. I. Germination course of Trichoderma viride. Conidia were incubated in germination medium at a concentration of $1 \times 10^{7} / \mathrm{ml}$. $O$, Percentage of swelling conidia as determined by uptake of cotton blue; $\triangle$, percentage of germinating conidia as observed in the light microscope; $\square$, conidium diameter $(\mu \mathrm{m})$ determined from electron micrographs.

Germ-tube emergence in the population commenced $9 \mathrm{~h}$ after inoculation. This period lasted until about the I 3 th hour, by which time $95 \%$ of the population had initiated germ tubes. The mean size of the mother conidia continued to increase during this period as well. The germination process occurred throughout the population in a semi-synchronous fashion and extended over a relatively long period of time. These characteristics help make Trichoderma a favourable organism for detailed study of conidium germination.

\section{Structural changes during conidium maturation and germination}

Structural changes occurring during the development of immature conidia through the resting, swelling and germinating stages were followed. Figs. 2 and 3 show the typical ultrastructural organization of immature, resting, swollen and germinated conidia. We can give no information concerning ribosomes and the internal structure of the nucleoplasm owing to the use of permanganate in the fixation procedure.

Mitochondria. The number, shape and some physical properties of mitochondria from maturing, resting, swollen and germinated conidia were measured.

Table I presents the number of mitochondrial figures per conidial section during the various developmental stages. There was an increase in the number of mitochondrial figures per section as the nascent conidia matured and entered the resting stage. After the resting conidia were inoculated into germination medium a $4 \mathrm{~h}$ period, analogous to the lag in conidial swelling (Fig. I), ensued during which there was no significant change in mitochondrial number. As observable swelling commenced, there was a marked increase in the number of mitochondrial figures and they continued to increase during the germ-tube emergence stage.

The question arose whether the multiplication of mitochondrial figures per section during germination was the result of the formation of one or a few highly branched organelles or of an actual increase in the number of individual mitochondria. To determine this point serial 
Table 1. Mitochondrial figures/conidial section during maturation, swelling and germination of Trichoderma viride

$\begin{array}{ccccc}\begin{array}{c}\text { Time of } \\ \text { incubation* } \\ \text { (h) }\end{array} & \begin{array}{c}\text { No. of } \\ \text { conidial } \\ \text { sections } \\ \text { examined }\end{array} & \begin{array}{c}\text { Percentage } \\ \text { swollen }\end{array} & \begin{array}{c}\text { Percentage } \\ \text { germinated }\end{array} & \begin{array}{c}\text { No. of } \\ \text { mitochondrial } \\ \text { figures section }\end{array} \\ \begin{array}{c}\text { Immature } \\ \text { o }\end{array} & 30 & - & - & 2.1 \\ 4 & 34 & 0 & 0 & 3.5 \\ 7 & 9 & 0 & 0 & 3.7 \\ 9 & 8 & 16 & 0 & 7 \cdot 0 \\ \text { II } & 20 & 83 & 0 & 9 \cdot 1 \\ \text { I3 } & 31 & 99 & 1 & 10 \cdot 0 \\ & \text { I6 } & - & 56 & 16.0\end{array}$

* Resting conidia ware incubated in garmination medium for the times indicated. The swelling process in this expəriment took somewhat longer than usual (compare with Fig. I).

sections of resting and germinated conidia were prepared from which three-dimensional models of the mitochondrial population at the two stages were constructed. Fig. 4 shows that in germinating conidia of Trichoderma, there is a sharp increase in the actual number of mitochondria.

Some of the mitochondria in nascent, immature conidia were relatively large and had convoluted shapes (Fig. 2a). Those of the mature, resting conidia were more regular in their contour, being ovoid or somewhat elongated (Fig. $2 b$ ). During the first $4 \mathrm{~h}$ after inoculation in germination medium (the lag period) the mitochondria began to change shape. Several mitochondria enlarged and assumed a dumb-bell configuration. As incubation continued there was a sharp rise in the number of dumb-bell and ring-shaped sections of mitochondria per conidium (Figs. $2 c$ and 5 ). The increased presence of this configuration accompanied the increase in mitochondrial number shown in Table I. The marked morphological changes which were occurring in the mitochondria during the early stages of germination can be visualized from the three-dimensional models (Fig. 4). The regular ovoid organelles of the resting conidia have developed into highly convoluted and cup-shaped ones.

Table 2 summarizes the data obtained for the various mitochondrial parameters measured. As can be seen, maturation and germination of $T$. viride conidia were accompanied by an increase in mean mitochondrial number, volume and surface area. The number of mitochondria per conidium was obtained by the stereological method (Weibel \& Gomez, 1962; Weibel et al. 1966). However, lack of geometrically well-defined shapes for the mitochondria at certain stages (see Fig. 4) posed a difficulty. This was partially overcome by empirical means using the three-dimensional models prepared from serial sections. The values obtained are therefore only approximate. As indicated, the ratio of mitochondrial surface area to volume $\left(S_{r} / V_{r}\right)$ remained constant throughout all the developmental stages (see Discussion).

Nucleus. The immature and resting conidia of $T$. viride contained a single nucleus which was oval in section and enveloped in a typical nuclear double membrane with pores (Fig. $2 a$, $b$ ). The nucleus did not change morphologically until about the 8th hour of incubation (late swelling stage). At this stage it acquired an irregular shape (Fig. 6), indicating the onset of division, and proceeded to divide before germ-tube emergence. After emergence of the germ tube the nuclei continued to divide while still in the mother conidium (Figs. 3 and $7 a$ ). One or more nuclei then migrated into the growing germ tube before formation of the first septum (Fig. 7 b). After septa formation nuclear division continued in the still active mother 

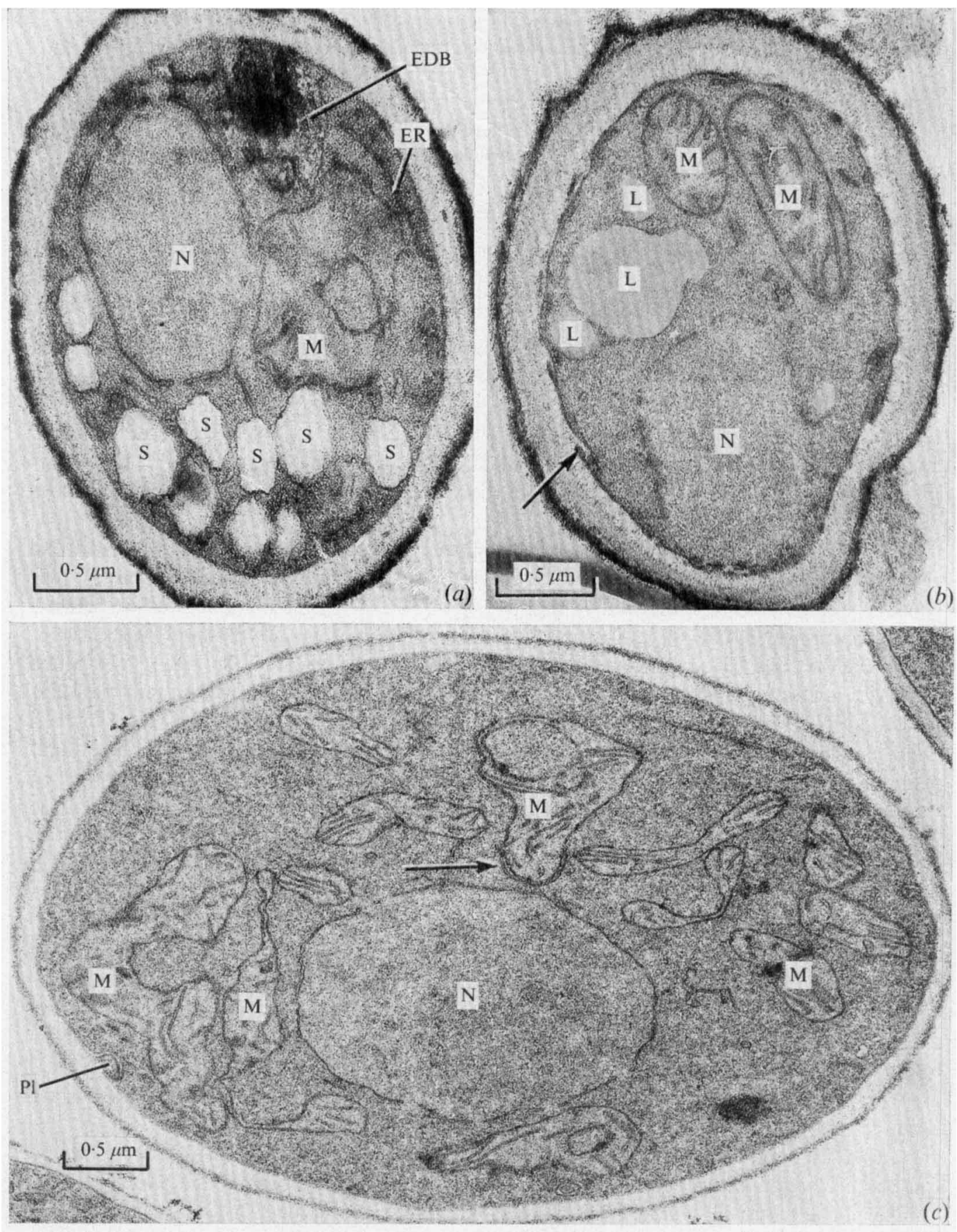

Fig. 2. (a) Immature conidium showing well-developed endoplasmic reticulum, large mitochondrion, stellate granules, electron-dense body and nucleus. (b) Resting conidium showing oval and elongated mitochondria, lipid granules and nucleus. Notice the invaginated plasmalemma (arrow). (c) A swollen conidium after $8 \mathrm{~h}$ incubation. The number of mitochondria has increased and many of them are dumb-bell shaped or convoluted. Endoplasmic reticulum is seen partially enveloping one of the mitochondria (arrow). A plasmalemmasome is shown (lower left). Abbreviations: ER, endoplasmic reticulum; M, mitochondrion; S, stellate granule; EDB, electron dense body; N, nucleus; L, lipid granule; Pl, plasmalemmasome; R, ring-like cisternae of endoplasmic reticulum. 


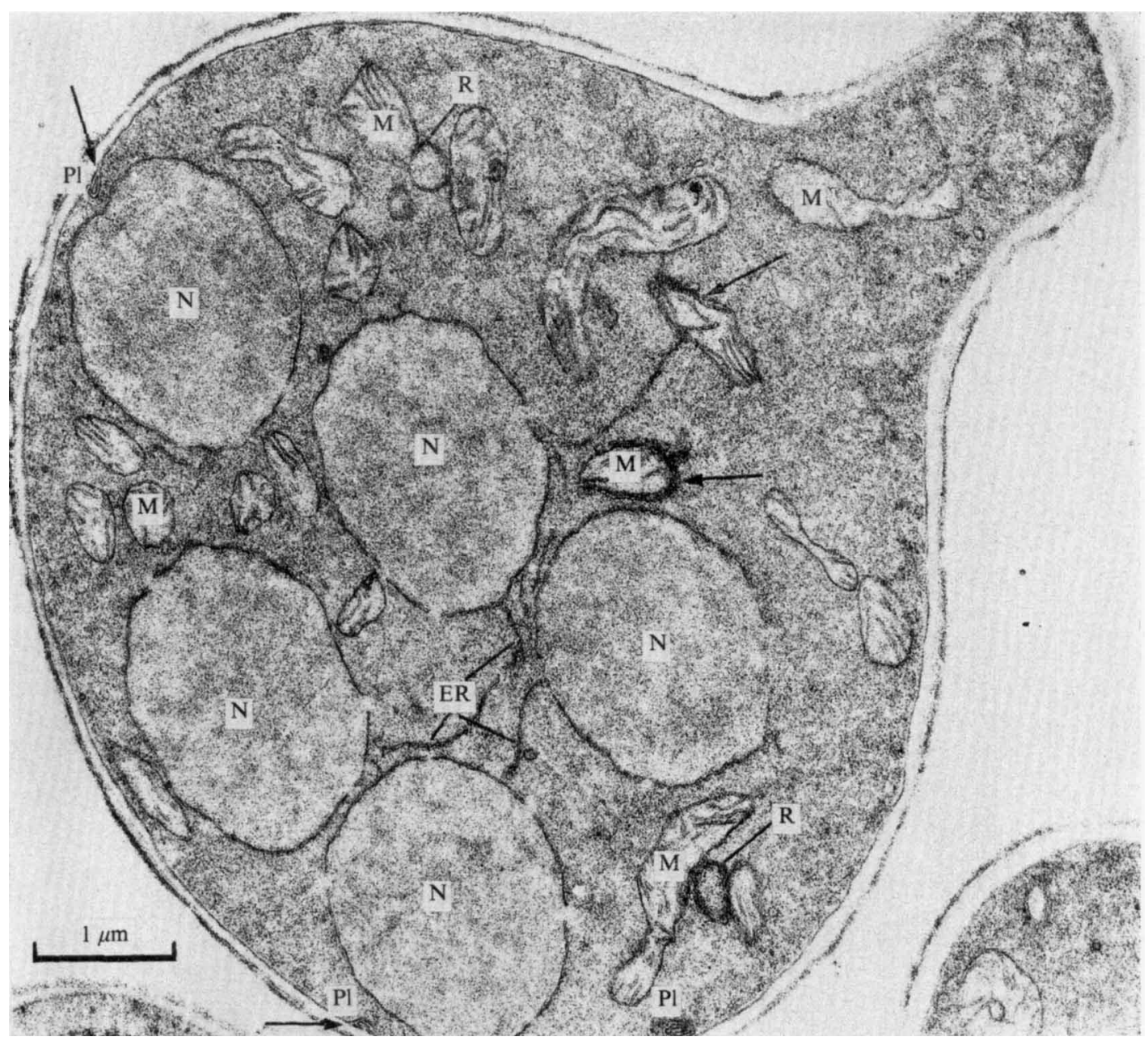

Fig. 3. A germinated conidium after $\mathrm{I} 3 \mathrm{~h}$ incubation. The nucleus has divided several times. Two mitochondria are partially enveloped by endoplasmic reticulum cisternae (arrows). Two pairs of mitochondria are linked by ring-like cisternae of endoplasmic reticulum. Three plasmalemmasomes are situated at the periphery; note continuity with the plasmalemma (arrows). For abbreviations, see Fig. 2.

conidium (Fig. $7 \mathrm{c}$ ). During all these stages of development, total nuclear volume remained at about $15 \%$ of cellular volume.

Plasmalemma and plasmalemmasomes. The plasmalemma of immature $T$. viride conidia was smooth (Fig. $2 a$ ) while that of the mature, resting conidia had invaginations (Fig. $2 b$ ) which can also be detected by the freeze-etching technique (Jones, I97I). Similar invaginations have been reported for many other fungi (Sassen, Remsen \& Hess, I967; McCoy et al. 1971 : Richmond \& Pring, 1971). During swelling and germ-tube emergence, these invaginations disappeared (Figs. $2 c$ and 3 ), possibly because of stretching of the membrane in response to an increased cytoplasmic volume.

In some immature conidia and in most swollen and germinated conidia of Trichoderma, membraneous structures can be seen along the periphery of the plasmalemma, which have 

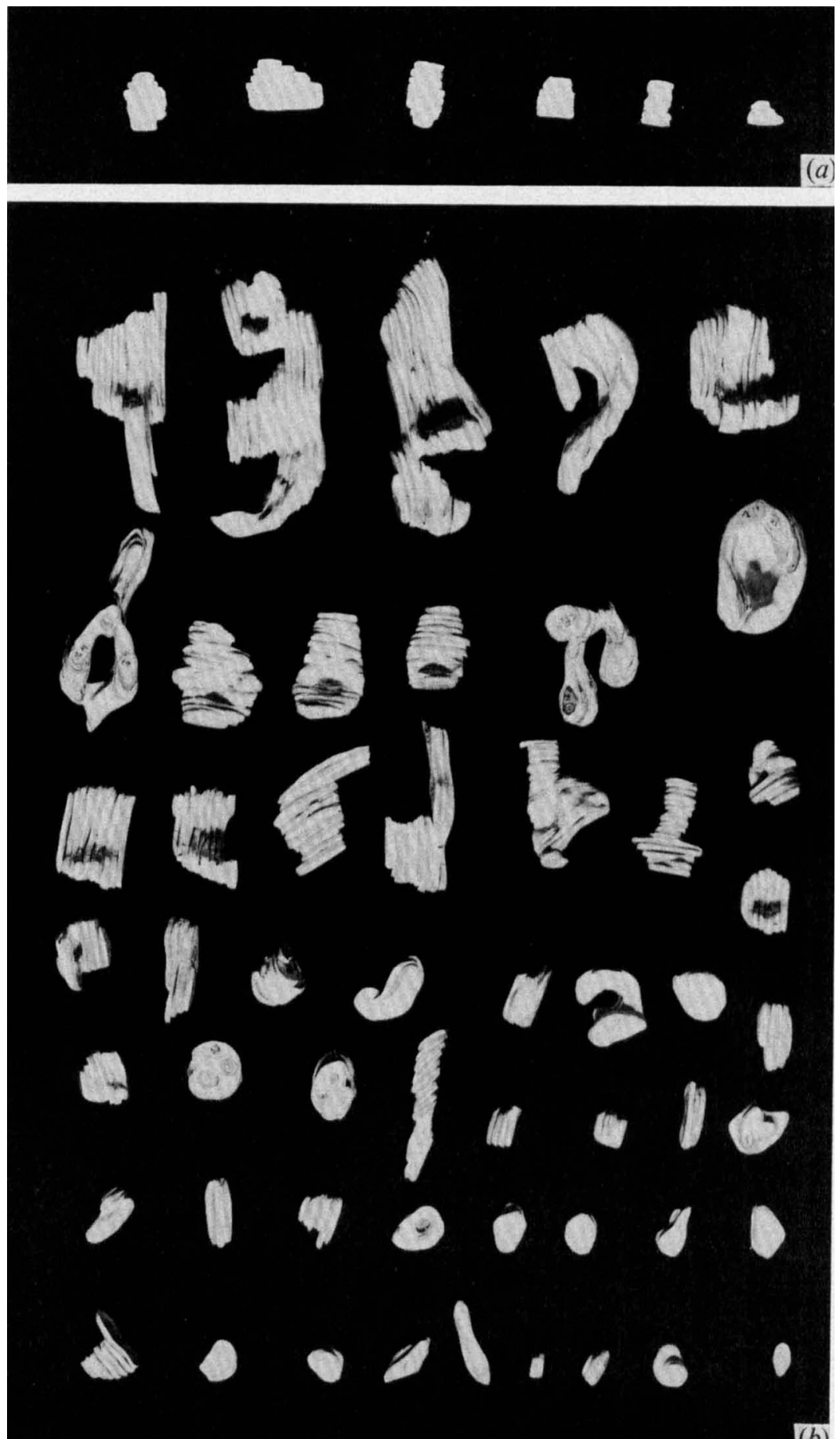

Fig. 4. (a) Three-dimensional reconstructions of the complete mitochondrial population taken from a serially sectioned resting conidium. (b) Three-dimensional reconstruction of the mitochondrial population taken from a serially sectioned germinated conidium after $13 \mathrm{~h}$ incubation. Not all the mitochondria are completely sectioned and probably not all of those actually existing in the conidium are presented here, since only $75 \%$ of the conidium was serially sectioned and reconstructed. Notice the convoluted cup-shaped organelles. The magnification factors in $(a)$ and $(b)$ are the same. 

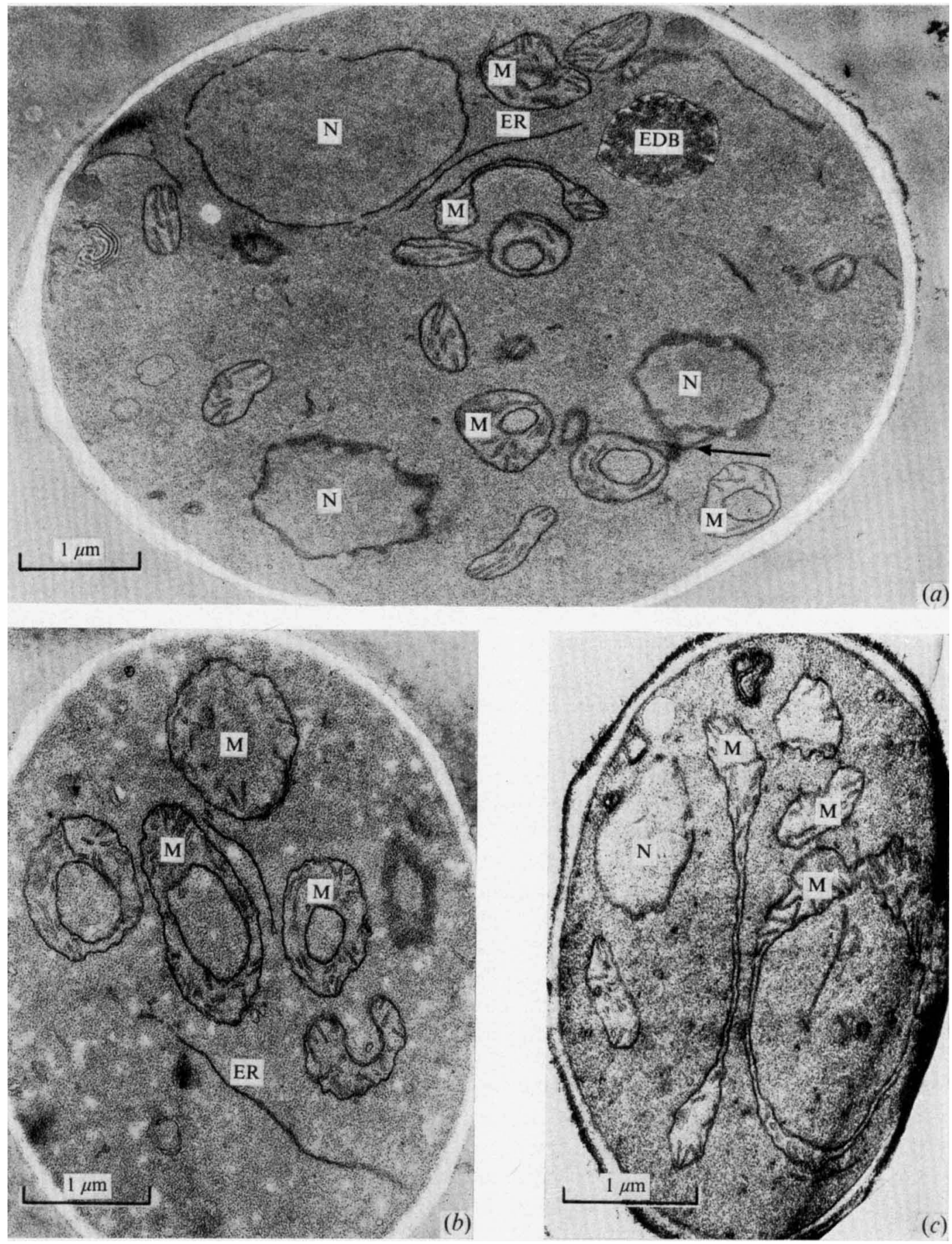

Fig. 5. (a) Germinated conidium after $\mathrm{I} 3 \mathrm{~h}$ incubation, showing a high proportion of dumb-bell- and ring-shaped mitochondrial profiles. Note endoplasmic reticulum interconnecting between mitochondrion and nucleus (arrow). (b) A swollen conidium after $8 \mathrm{~h}$ incubation showing a predominance of ring-shaped mitochondrial figures. (c) A swollen conidium from the same population as $(b)$, showing transections of giant cup-shaped mitochondria. For abbreviations, see Fig. 2. 


\section{Table 2. Mitochondrial structural parameters during maturation and germination of Trichoderma viride}

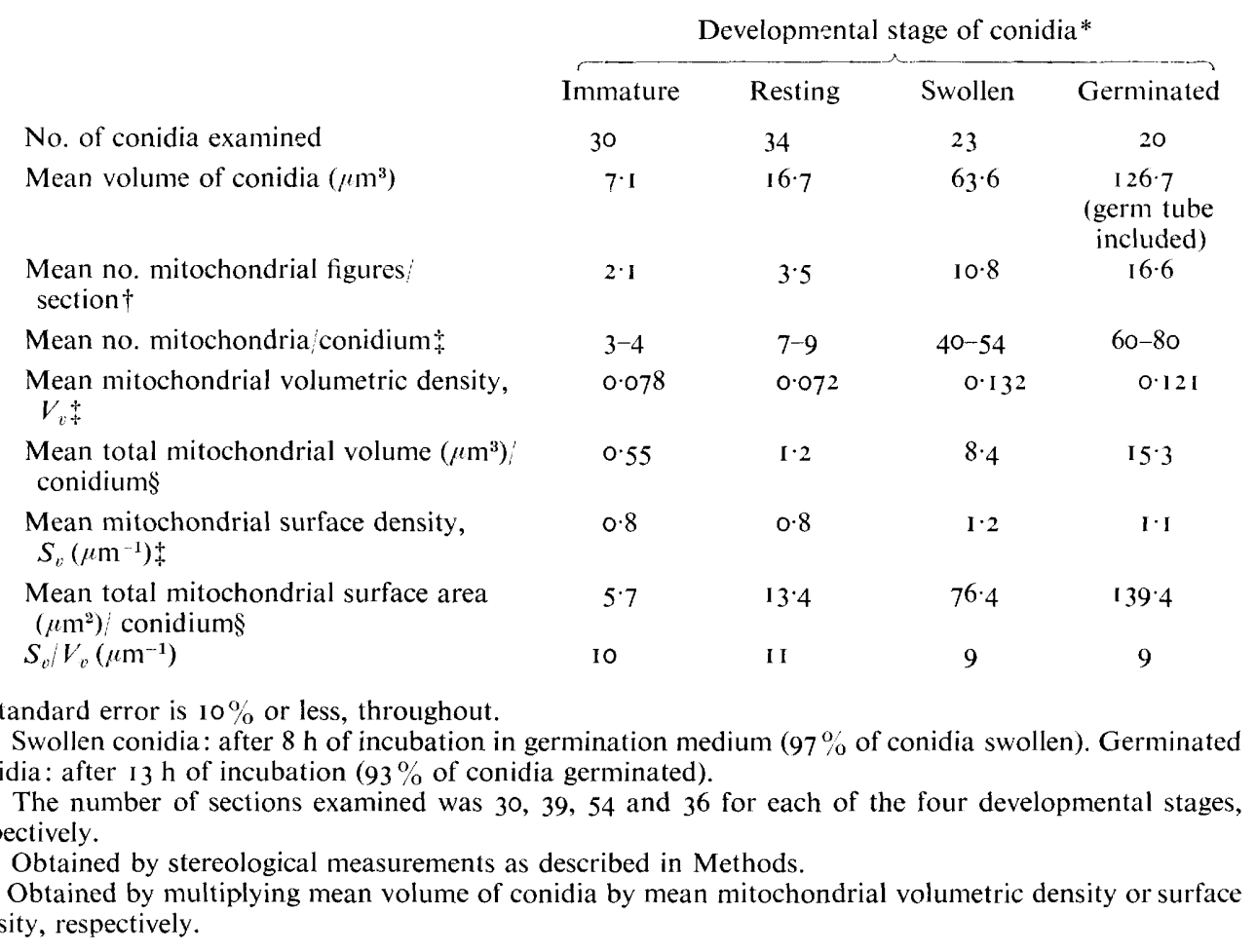

been designated plasmalemmasomes (Marchant \& Robards, 1968). Such structures have not been found in resting conidia. The plasmalemmasomes in $T$. viride are typically built of spiral infoldings of the plasmalemma (Fig. $8 a$; see also Figs. $2 c, 3,6 a, 7 a, c$ and 11 ). The membranes rarely appear as vesicles in section (Fig. $8 c$ ). These structures are randomly situated at the periphery of the plasmalemma and are not confined preferentially to any one site. Their structural characteristics and their appearance at a very active stage of development might imply a role in increasing the surface area of the plasmalemma.

Inclusion bodies. Two types of inclusion bodies were present in the immature conidium: electron-dense bodies which in some conidia were partially evacuated, and stellate vacuoles which were randomly scattered in the cytoplasm (Fig. $2 a$ ). The stellate vacuoles sometimes contained granular material. These two types of membrane-enclosed bodies were absent from resting conidia, which possessed only lipid granules (Fig. $2 b$ ) usually situated at the periphery of the cell. These lipid bodies, which did not have limiting membranes, disappeared gradually during the swelling process and were rarely found in germinated conidia. Within $2 \mathrm{~h}$ after incubation in germination medium, the electron-dense bodies reappeared (Fig. 9) indicating ultrastructural and biochemical changes during the lag period. At this early stage the bodies were filled with electron-opaque material. During conidium swelling and germination these bodies became partially evacuated (Figs. 6a, 7 and 11 ) and when the germ tube emerged they migrated into it (Fig. $7 b, c$ ). From their staining properties and morphological characteristics the electron-dense bodies are thought to contain phospholipoproteins (Buckley, Sjaholm \& Sommer, I966). 

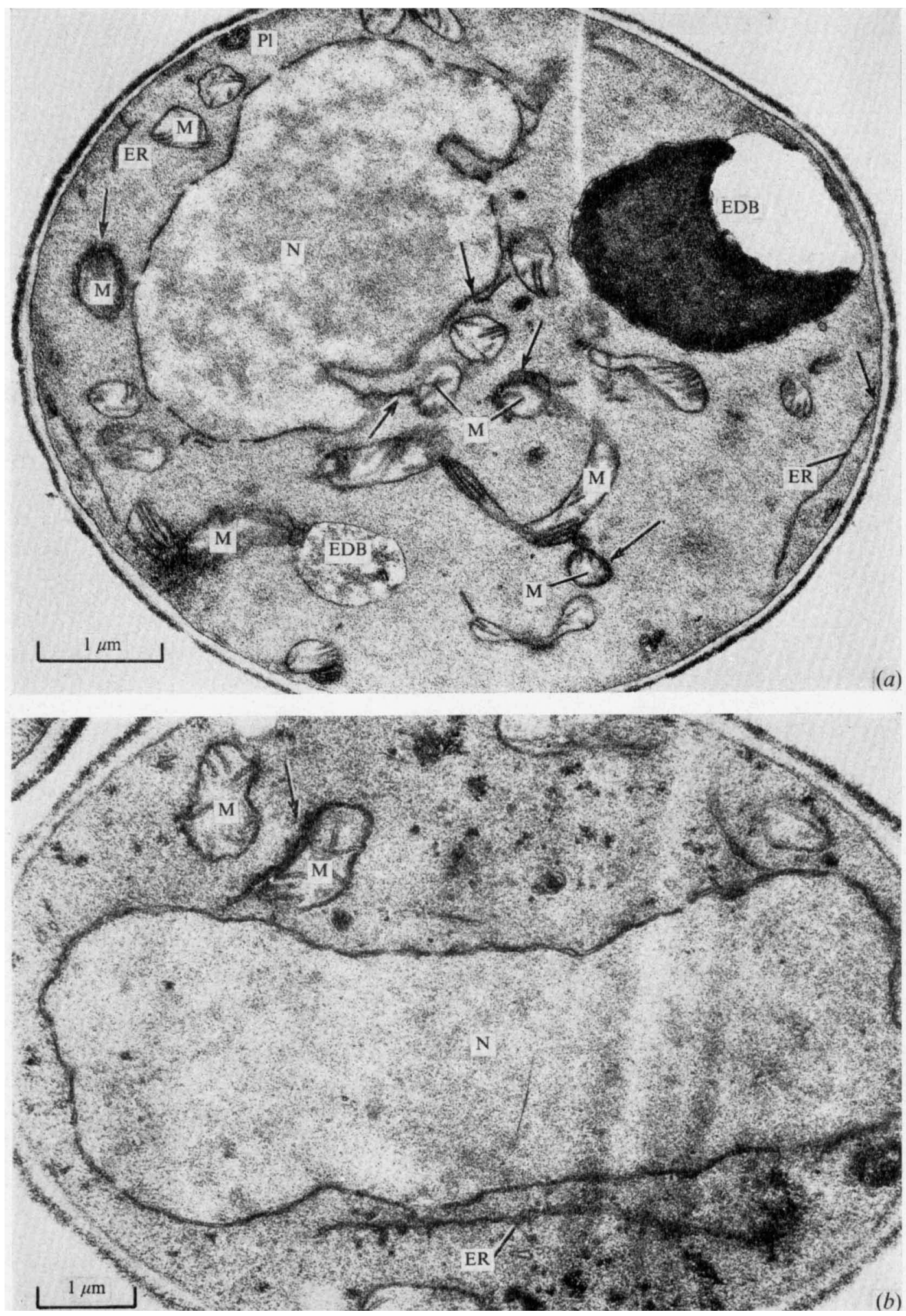

Fig. 6. Conidia after $8 \mathrm{~h}$ incubation in germination medium (late swelling stage). (a) Incipient fission of enlarged nucleus. Note close association of endoplasmic reticulum with mitochondria, nucleus and plasmalemma and the apparent connexion between the nucleus and a mitochondrion (arrows). (b) Elongated nucleus in the process of division. Note mitochondrion partially enveloped by endoplasmic reticulum (arrow). For abbreviations, see Fig. 2 . 


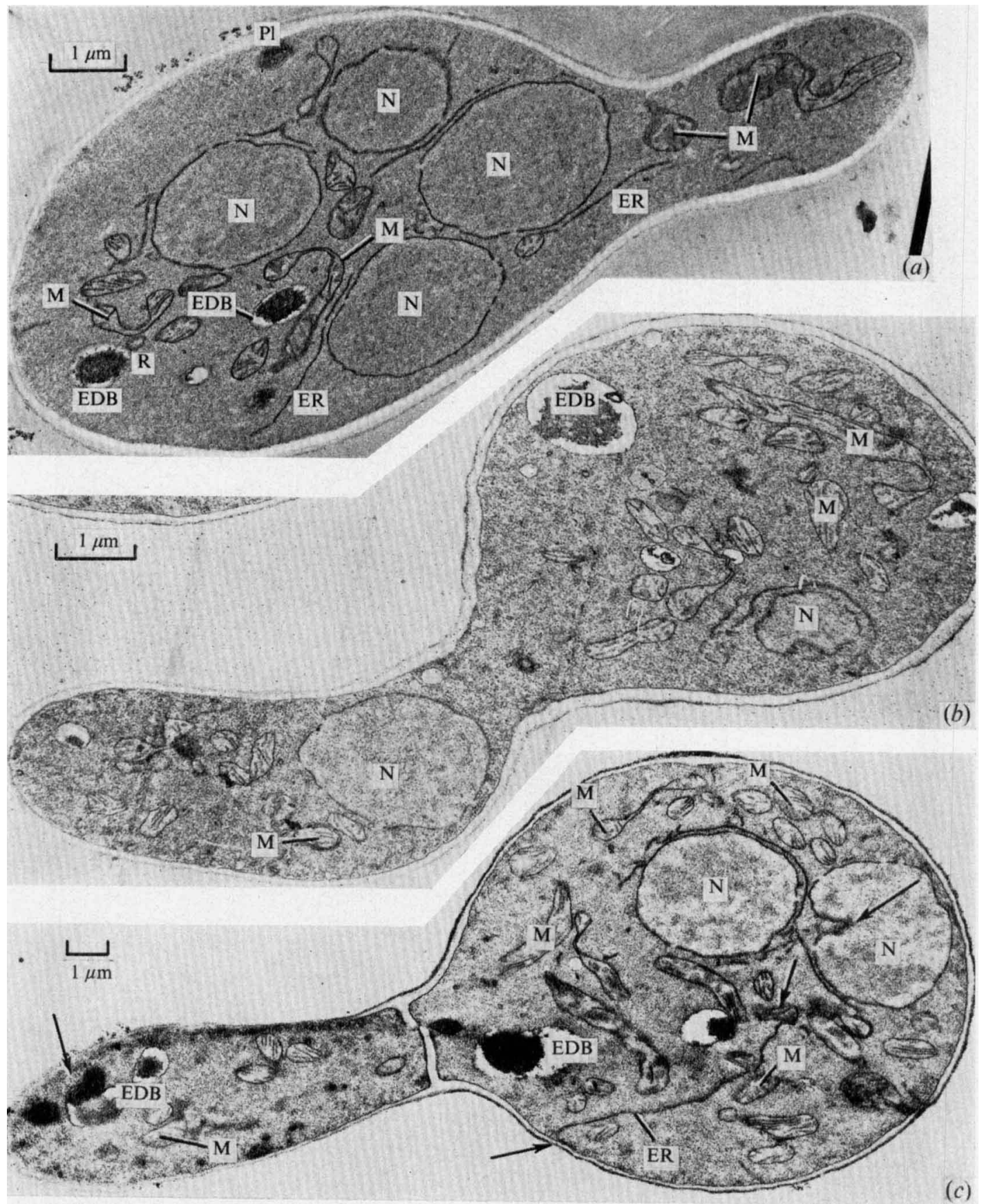

Fig. 7. Germinated conidia after $\mathrm{I} 3 \mathrm{~h}$ incubation. (a) Four nuclei are seen in the mother conidium before migration into the germ tube begins. Note the dumb-bell-shaped mitochondria and the partially evacuated electron dense bodies. $(b)$ One nucleus has migrated into the newly formed germ tube. (c) A conidium after the formation of the first septum. A nucleus is undergoing fission while still in the mother conidium (arrow). The electron-dense bodies are partially evacuated and a few are seen inside the germ tube. Note a close association between plasmalemmasome and electron-dense body at the tip of the germ tube, and between endoplasmic reticulum and plasmalemma (arrows). In serial sections this same endoplasmic reticulum profile is seen to interconnect between the plasmalemma and the mitochondrion, indicated by an arrow. For abbreviations, see Fig. 2. 

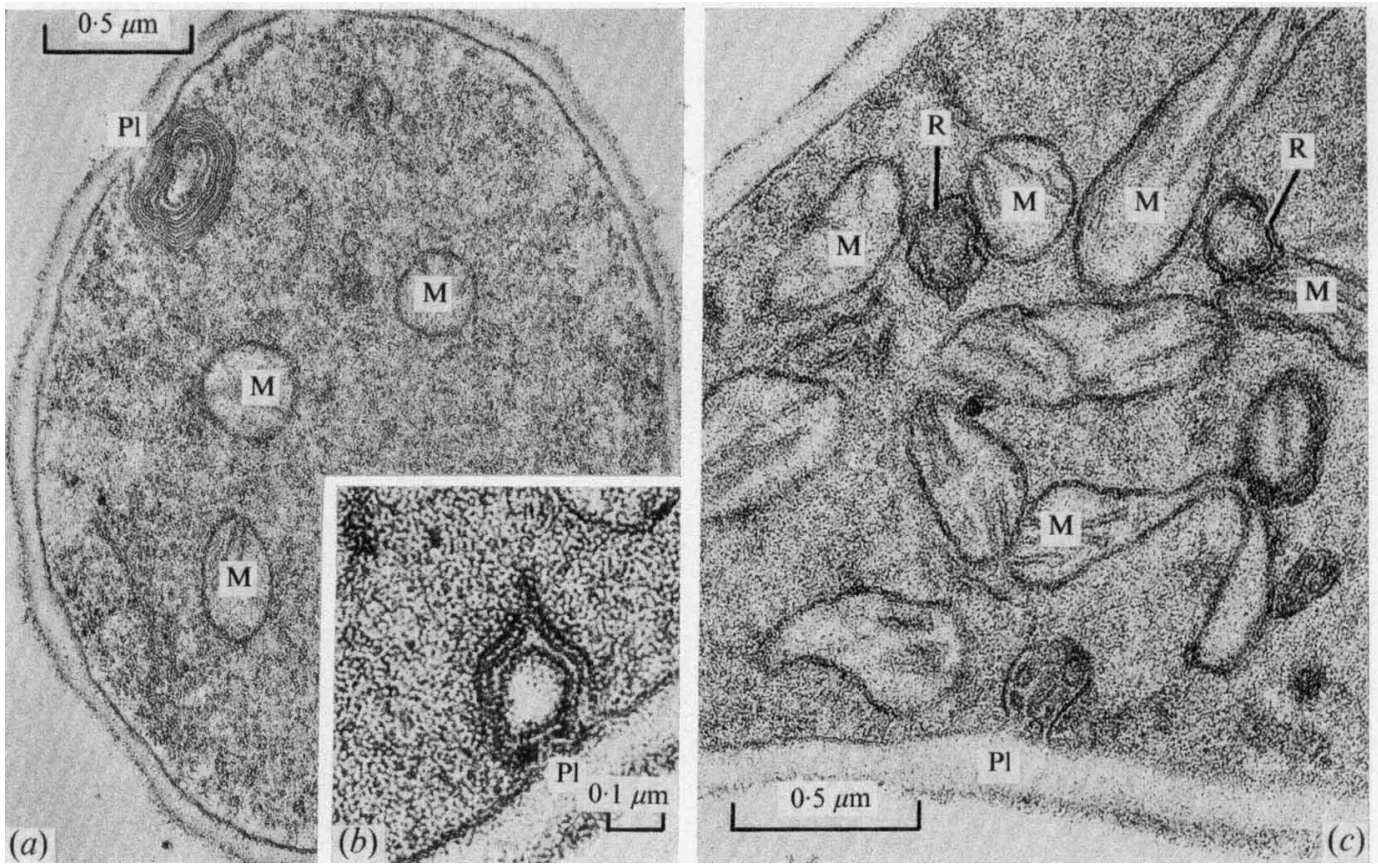

Fig. 8. (a) A typical spiral plasmalemmasome from a swollen conidium. Note the continuity with the plasmalemma. (b) A plasmalemmasome from a serial section of the conidium shown in Fig. 3. Note the less electron-dense centre. (c) A vesicular-type plasmalemmasome from a germinated conidium ( $13 \mathrm{~h}$ incubation) which is continuous with the plasmalemma. Two ring-like endoplasmic reticulum cisternae, each situated between neighbouring mitochondria, are seen. For abbreviations, see Fig. 2.

Endoplasmic reticulum. Marked changes occurred in the endoplasmic reticulum during conidium ontogeny. The immature conidia possessed a relatively large amount of endoplasmic reticulum with long profiles sometimes extending throughout the whole conidium (Fig. IO). These gradually disappeared during the maturation process, resulting in a resting conidium without any endoplasmic reticulum or, rarely, with sparse, short profiles (Fig. $2 b$ ). During the first $4 \mathrm{~h}$ in germination medium (lag period) a few short membrane segments reappeared, randomly scattered in the cytoplasm. During the swelling and germination processes the content of endoplasmic reticulum gradually increased (Figs. 2c, 3, 6 and 7). Continuity between the endoplasmic reticulum and the mitochondria, nucleus and plasmalemma has been reported in several organisms (Bracker \& Grove, I97I ; Franke \& Kartenbeck, 1971; Gull \& Trinci, 197I; Morré, Merritt \& Lembi, I97I). During swelling and germ-tube emergence in Trichoderma a close association could often be seen between the endoplasmic reticulum and these same organelles (see Fig. $6 a$ and Figs $2 c, 3,5 a, 6 b, 7 b, c$, $8 c$ and $\mathrm{I}$ I). In addition, a close association with plasmalemmasomes and electron dense bodies also occurred in this system (Fig. II). As indicated by Figs. 3, 5a, 6a, $7 c$ and I I $b, c$ the endoplasmic reticulum may, in fact, have been playing a role in connecting the different organelles during the germination process.

Conidium wall. The conidium wall has been described in many ultrastructural studies dealing with fungal spore germination. Consequently, we shall emphasize only those features which pertain to conidium maturation and germination. The walls of immature 

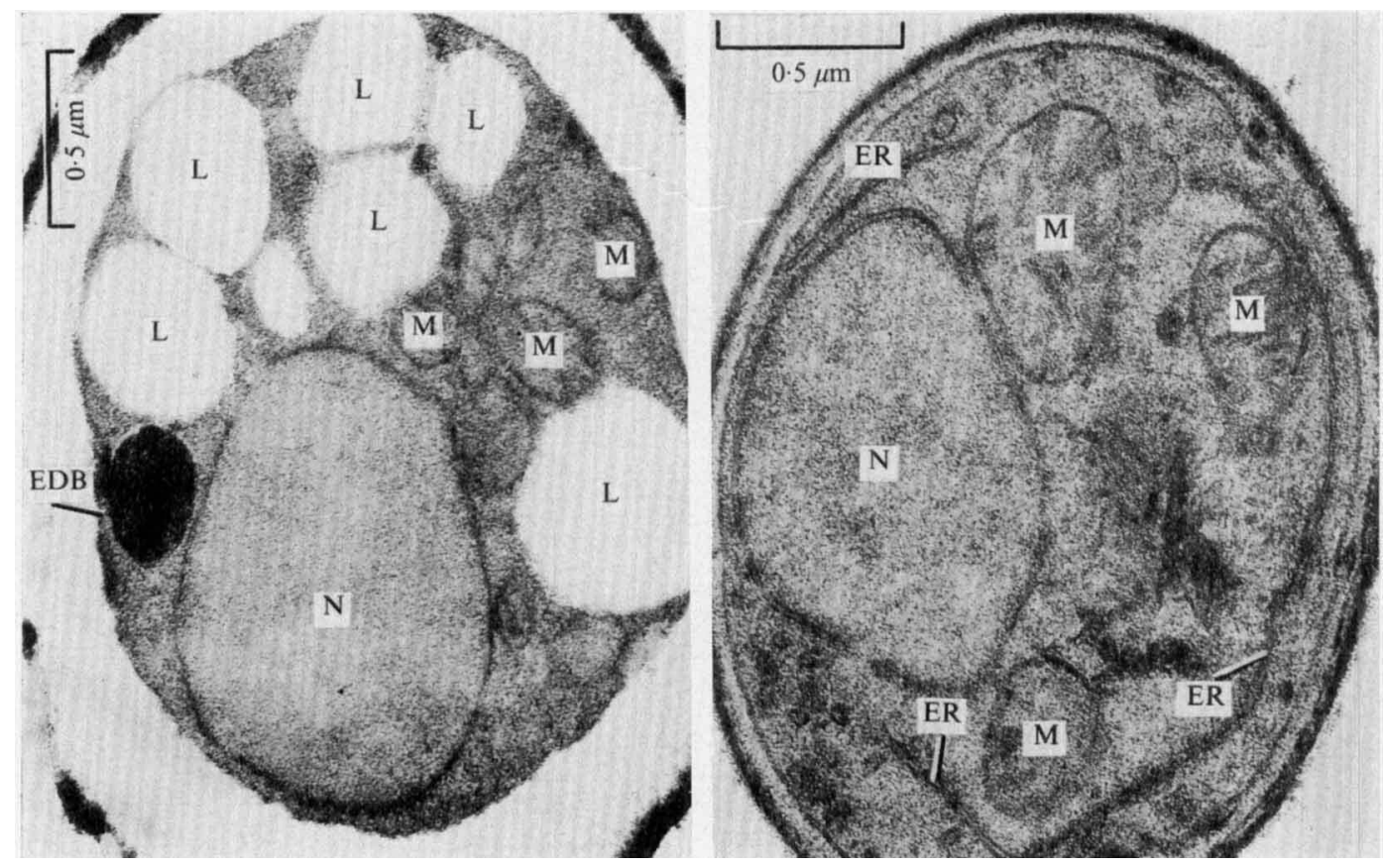

Fig. 9. (a) A conidium after $2 \mathrm{~h}$ incubation in germination medium. An electron-dense body is seen together with lipid granules. This developmental stage of the conidium is very difficult to process and section; note the thick cell wall. For abbreviations, see Fig. 2.

Fig. 10. Immature conidium showing well-developed endoplasmic reticulum profiles extending the length of the cell. For abbreviations, see Fig. 2.

conidia were thinner than those of resting conidia, occupying $25 \%$ of the conidial volume as opposed to $34 \%$ at the resting stage. As conidium swelling commenced, the wall stretched and became thinner once again. Upon emergence of the germ tube the outer wall layer was ruptured and the germ-tube wall appeared continuous with the inner layer of the mother conidium wall (Figs. 3 and 7 ). In swollen and germinated conidia the wall occupied $11 \%$ of the total volume.

The immature conidium wall was permeable to fixatives and other reagents used in preparation for electron microscopic observation. However, after conidium maturation and during the first hour of incubation in germination medium, the wall was impermeable to these materials. This difficulty was overcome by cracking the resting conidia in the presence of fixatives with a Braun homogenizer (see Methods). Two hours after inoculation in germination medium the conidium wall became permeable once again, thereby indicating metabolic changes during this period.

\section{Maturation}

DISCUSSION

Conidium maturation in fungi has not been as intensively studied as has the germination process. In Aspergillus nidulans the spore wall was reported to be reduced in thickness during maturation (Oliver, 1972) while in Rhizopus nigricans and $R$. sexualis it apparently becomes thicker (Hawker \& Abbott, 1963). Other changes which were noted include the addition 

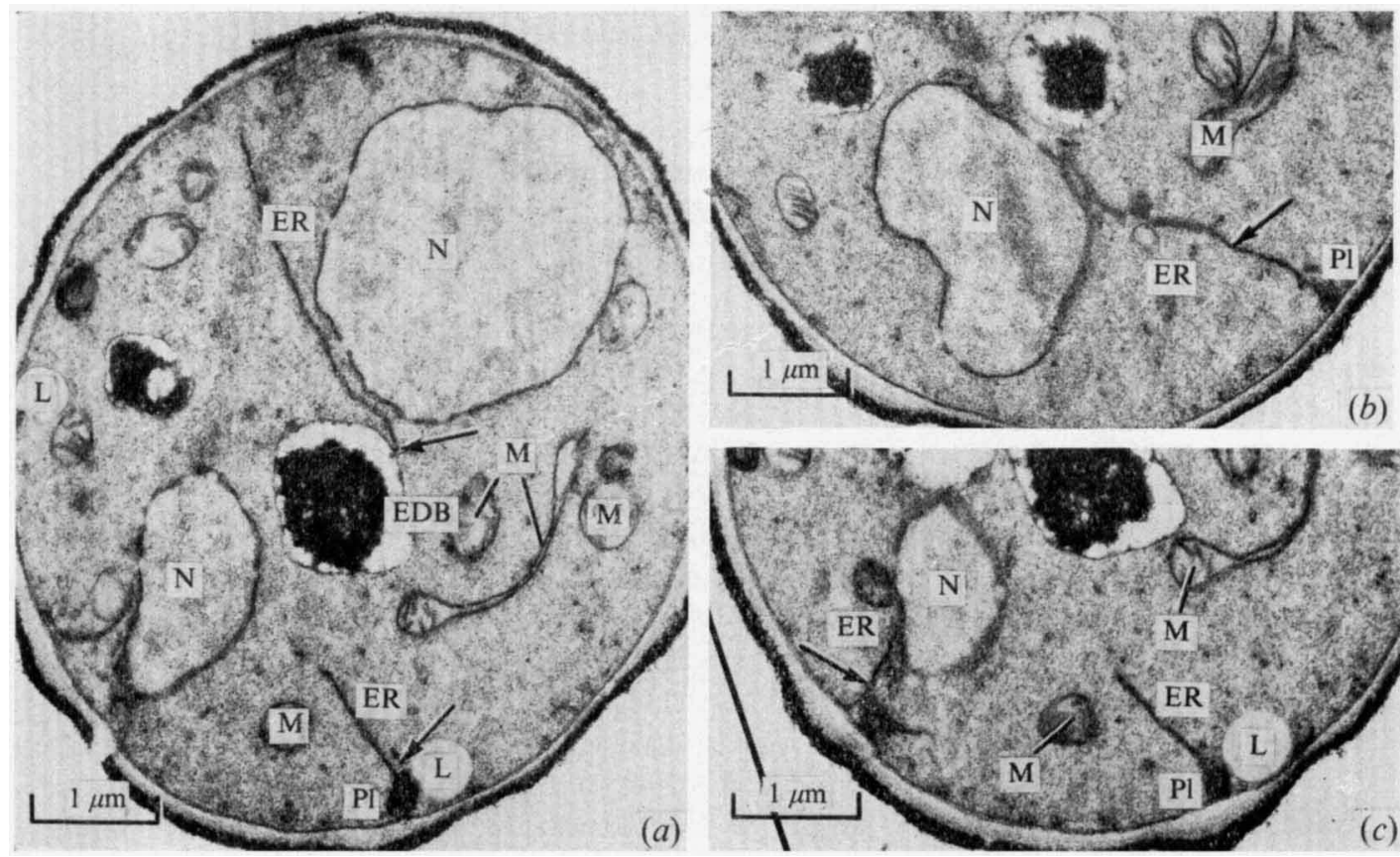

(b)

(a)

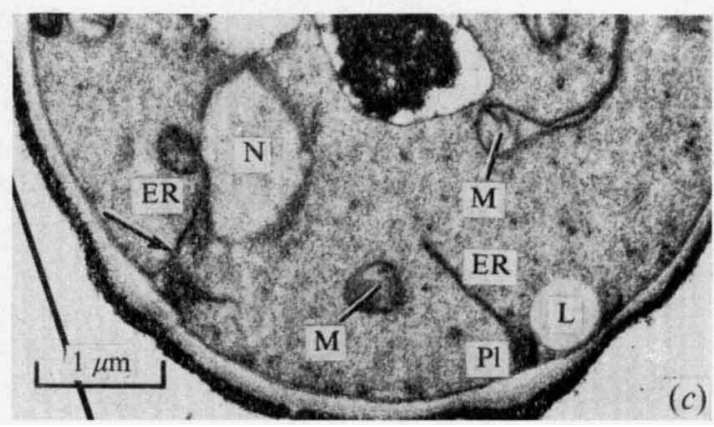

Fig. I I. Non-consecutive serial sections of a germinated conidium after $13 \mathrm{~h}$ incubation, showing connexions between the endoplasmic reticulum and various internal structures. In each section a connexion can be seen between the endoplasmic reticulum and a particular plasmalemmasome [see lower right-hand corners, and arrow in $(a)$ ]. Note the connexion between the endoplasmic reticulum and an electron-dense body (arrow) in (a), the endoplasmic reticulum interconnecting between plasmalemmasome and nucleus (arrow) in $(b)$, and another endoplasmic reticulum profile in $(c)$ interconnecting, in this case, between the plasmalemma and this same nucleus (arrow). For abbreviations, see Fig. 2.

of a third layer in the conidial wall (Weisberg \& Turian, 1971), a decrease in the endoplasmic reticulum content (Weisberg \& Turian, 1971), increase in mitochondrial size and structural complexity (Hawker, 1966), a possible division of mitochondria (although no conclusive quantitative data was given) (Weisberg \& Turian, I971), and changes in the shape and size of the conidiospore (Trinci, Peat \& Banbury, 1968).

In Trichoderma viride we observed several ultrastructural developments during maturation of the conidium: (i) There was a $13 \%$ increase in conidium diameter which corresponded to a $135 \%$ increase in volume (Table 2). (ii) The wall became thicker and impermeable to cotton blue and fixatives. (iii) The smooth outline of the plasmalemma acquired the typical infoldings found in the resting conidium (see Fig. $2 a, b$ ) and the plasmalemmasomes found in some immature conidia disappeared. (iv) The well-developed endoplasmic reticulum present in the immature conidium (Figs. $2 a$ and 10) disappeared (Fig. $2 b$ ). (v) The large cup-shaped mitochondria sometimes seen in the young conidia became smaller and ellipsoidal and the number of mitochondria increased during the maturation process (Tables I and 2). Since the cup-shaped mitochondria reappeared at the time of active mitochondrial division during germination (see below), their disappearance during conidial maturation can be understood in terms of the overall diminution of synthetic activity in conidia as they enter the resting stage. (vi) The electron-dense bodies found in the immature conidia degenerated and were usually not present in resting conidia. (vii) Finally, the stellate bodies 
disappeared while lipid granules, typical of the resting conidium, appeared (Fig. $2 a, b$ ). The chemical composition of these bodies in the immature conidium is unknown. However, from the examination of many micrographs, there seems to be a gradual transition from the stellate bodies of very young conidia to a more lipid type of granule in more mature (but not fully matured) conidia. This suggests that during maturation the stellate granules underwent changes and became the typical lipid granules of the resting conidium.

\section{Germination}

Mitochondria. A striking structural change in the mitochondria during conidium maturation and germination was the disappearance of the dumb-bell and ring-shaped configurations at the resting conidium stage and their reappearance during conidial swelling. Similar mitochondrial shapes have been found in a number of other organisms under various physiological conditions (Christensen \& Chapman, I959; Bell \& Mühlethaler, I962; Weiss, I963; Stephens \& Bils, 1965; Buckley et al. 1966; Hawley \& Wagner, 1967; Bagshaw, Brown \& Yeoman, 1969) where they were sometimes interpreted as being mitochondrial division figures (Hawley \& Wagner, I967; Mitchell \& McKeen, 1970). Examination of serial sections of such configurations in $T$. viride, however, revealed that the dumb-bell and ring shapes were derived from slicing a three-dimensional cup-shaped organelle (Fig. $4 b$ ). Thus, while there may be a relation between the dumb-bell and ring shapes seen in section and mitochondrial division as measured morphologically (Hawley \& Wagner, I967), the two-dimensional profile, in itself, is not a division figure. In Trichoderma there seems to be a correlation between the presence of dumb-bell and ring-shaped mitochondrial profiles and an increase in the absolute number of mitochondria. However, a distinctive division profile in this organism has not been elucidated. Possibly the fission process is so rapid that the probability of finding unequivocal division figures is fairly low.

The origin of the cup-shaped mitochondria is not completely understood. In swollen and germinated conidia of $T$. viride many of these mitochondria had increased in size (Figs. $4 b$ and $5 c$ ). This growth included biosynthesis of membranes and matrix. Our data (Table 2) show an increase in total mitochondrial surface area and in total mitochondrial volume during the germination process; however, the ratio of surface|area to volume $S_{v} / S_{v}$ remained constant. Since for a given shape the volume is proportional to the third power of a linear dimension and the surface area to the second power, if the mitochondrion grows while preserving its shape then the surface to volume ratio should decrease. The finding of a constant ratio indicates that the surface is growing relatively faster than the volume, this excess surface causing the formation of the convoluted shapes. The three-dimensional cup shape may then result from a rate of synthesis of the outer mitochondrial membrane which is not compensated for by an appropriate rate of matrix synthesis.

Plasmalemmasomes. Membranous structures associated with the plasmalemma were seen in some immature conidia. They redeveloped during the swelling process and were commonly seen at about $9 \mathrm{~h}$ after inoculation in germination medium; these are the plasmalemmasomes. The origin and function of these structures have been controversial ever since they were first reported by Girbardt ( 1958 ). The plasmalemmal origin of these membranes in $T$. viride is suggested from the continuity observed between the two structures (Figs. $2 c, 3$ and $8 a, c$ ). Likewise, the plasmalemmasomes in Figs. $2 c$ and 3, which were at their initial stages of development (based on serial sections), are seen to be continuous with the plasmalemma.

The plasmalemmasomes in Trichoderma differ in their architecture from those reported in other organisms. In section they appear as spiral infoldings of the plasmalemma (Figs. 3, $6 a, 7 a, 8 a$ and I $a, c)$; only infrequently did we find a structure which appeared filled with 
small vesicles (Fig. $8 c$ ) which might be interpreted as cross-sections of tubules. In most cases the central loop or loops embraced a matrix which was less electron dense than the surrounding cytoplasm (Fig. 8b).

An additional feature of plasmalemmasomes in germinating Trichoderma conidia which has not been reported for any other organism was their close association with the electrondense bodies (Fig. $7 c$ ) and their frequently observed connexion with the endoplasmic reticulum (Fig. 11). Together with the presence of an electron-transparent core (Fig. 8b) this suggests that the plasmalemmasomes in Trichoderma are involved in some sort of synthetic activity during germination.

Endoplasmic reticulum. Synthesis of endoplasmic reticulum during spore germination seems to be a widespread feature in fungi and may be correlated with the increase in synthetic activity of the spores (Lowry \& Sussman, I968; Fletcher, I97I ; Gull \& Trinci, I97I; Florance, Denison \& Allen, 1972). The endoplasmic reticulum in Trichoderma was in the form of sheets. They may be long sheets, as was found in immature (Fig. 10), swollen (Fig. 6b) and germinated (Fig. 7) conidia, or short sheets, ramifying membranes and rings of double membranes. These ring-like cisternae were usually seen between neighbouring mitochondria (Figs. 3 and $8 \mathrm{c}$ ). Some endoplasmic reticulum sheets partially enveloped mitochondria and were often seen covering the wide ends of dumb-bell-shaped mitochondria (Figs. $2 c, 3,6$ and $7 b, c$ ). Double membranes, continuously enveloping mitochondria, were reported for sporangia of Gilbertella (Bracker, 1966) and for germinating spores of Botrytis cinerea (Gull \& Trinci, 197I). They were interpreted as autophagic vacuoles involved in mitochondrial degeneration. Such a function cannot be assigned in the case of $T$. viride, as there is no indication of any degenerating mitochondria during germination. On the contrary, the enveloping membranes may have been involved in mitochondrial biogenesis since they were found at a stage of active mitochondrial multiplication.

Electron-dense bodies. Electron-dense bodies similar to those described for Trichoderma have been found in resting and germinating conidia of Botrytis cinerea (Buckley et al. 1956) and Penicillium megasporum (Remsen, Hess \& Sassen, 1967). In all three organisms these vacuoles break down during germination into smaller units and their contents are gradually dissipated. Buckley et al. (1966) suggest that these bodies contain phospholipoprotein which may provide material for the assembly of membranous organelles during germination. The dissipation of these inclusions during germination and their possible connexion via the endoplasmic reticulum to other organelles in Trichoderma fits this assumption.

We gratefully acknowledge the assistance of Mrs M. Shamir and Miss A. Kogman. This study was supported in part by a U.S.D.A. grant under U.S. Public Law 480.

\section{REFERENCES}

Bagshaw, V., Brown, R. \& Yeoman, M. M. (I969). Changes in the mitochondrial complex accompanying callus growth. Annals of Botany 33, 34-54.

Bell, P. R. \& Mühlethaler, K. (I962). The fine structure of the cells taking part in oogenesis in Pteridium aquilinum (L) Kuhn. Journal of Ultrastructural Research 7, 452-466.

BRACKER, C. E. (1966). Ultrastructural aspects of sporangiospore formation in Gilbertella persicaria. In The Fungus Spore, pp. 39-60. Edited by M. F. Madelin. London: Butterworths.

Bracker, C. E. \& Grove, S. (1971). Continuity between cytoplasmic endomembranes and outer mitochondrial membranes in fungi. Protoplasma $73, \mathrm{I} 5-34$.

Buckley, P. M., SJaholm, V. E. \& SOmmer, N. F. (1966). Electron microscopy of Botrytis cinerea conidia. Journal of Bacteriology 9r, 2037-2044. 
Christensen, A. K., \& ChAPMAN, G. B. (1959). Cup-shaped mitochondria in interstitial cells of the albino rat testis. Experimental Cell Research $\mathbf{1 8}, 576-579$.

Cochrane, V. W. (1966). Respiration and spore germination. In The Fungus Spore, pp. 20I-215. Edited by M. F. Madelin. London: Butterworths.

Edelman, M., Verma, I. M., Herzog, R., Galun, E. \& Littauer, U. Z. (i97I I). Physico-chemical properties of mitochondrial ribosomal RNA from fungi. European Journal of Biochemistry 19, 372-378.

Ekundayo, J. A. \& Carlile, M. J. (1964). The germination of sporangiospores of Rhizopus arrhizus; spore swelling and germ-tube emergence. Journal of General Microbiology 35, 261-269.

Fletcher, J. (1971). Fine-structural changes during germination of conidia of Penicillium griseofulvum Dierckx. Annals of Botany 35, 44I-449.

Florance, E. R., Denison, W. C. \& Allen, T. C., Jun. (1972). Ultrastructure of dormant and germinating conidia of Aspergillus nidulans. Mycologia 64, 115-123.

Franke, W. W. \& Kartenbeck, J. (197I). Outer mitochondrial membrane continuous with endoplasmic reticulum. Protoplasma $\mathbf{7 3}, 34-5 \mathrm{I}$.

Galun, E. \& Gressel, J. (1966). Morphogenesis in Trichoderma: suppression of photoinduction by 5-fluorouracil. Science, New York 15I, 696-698.

Girbardt, M. (1958). Über die Substruktur von Polystictus versicolor L. Archiv fïr Mikrobiologie 28, 255-269.

Gottlieb, D. (1966). Biosynthetic processes in germinating spores. In The Fungus Spore, pp. 217-233. Edited by M. F. Madelin. London: Butterworths.

Gressel, J. \& Galun, E. (1967). Morphogenesis in Trichoderma: photoinduction and RNA. Developmental Biology 15, 575-598.

Gull, K. \& Trinci, A. P. J. (1971). Fine structure of spore germination in Botrytis cinerea. Journal of General Microbiology 68, 207-220.

Hawker, L. E. (1966). Germination: morphological and anatomical changes. In The Fungus Spore, pp. I5 I163. Edited by M. F. Madelin: London, Butterworths.

HAWkER, L. E. \& AввотT, P., MCV. (I963). An electron microscope study of maturation and germination of sporangiospores of two species of Rhizopus. Journal of General Microbiology 32, 295-298.

Hawker, L. E., Thomas, B. \& Beckett, A. (1970). An electron microscope study of structure and germination of conidia of Cunninghamella elegans Lendner. Journal of General Microbiology 60, 18 I-189.

Hawley, E. S. \& WAGNer, R. P. (1967). Synchronous mitochondrial division in Neurospora crassa. Journal of Cell Biology 35, 489-499.

JonEs, D. (197I). Frozen-etched spores of Trichoderma viride. Transactions of the British Mycological Society 57, 348-351.

KumAGAI, T. \& ODA, Y. (I969). An action spectrum for photoinduced sporulation in the fungus Trichoderma viride. Plant and Cell Physiology 1o, 387-392.

KüNTZEL, H. (1971). The genetic apparatus of mitochondria from Neurospora and yeast. Current Topics in Microbiology and Immunology 54, 94-I 18.

Linnane, A. W., Haslam, J. M., Lukins, H. B. \& Nagley, P. (1972). The biogenesis of mitochondria in microorganisms. Annual Review of Microbiology 26, 163-198.

LOWRY, R. J. \& SusSMAN, A. S. (1968). Ultrastructural changes during germination of ascopores of Neurospora tetrasperma. Journal of General Microbiology 5I, 403-409.

Marchant, R. \& Robards, A. W. (I968). Membrane systems associated with the plasmalemma of plant cells. Annals of Botany 32, 457-47I.

Martin, J. F. \& Nicolás, G. (1970). Physiology of spore germination in Penicillium notatum and Trichoderma lignorum. Transactions of the British Mycological Society 55, I4I-I48.

McCoy, E. C., Girard, A. E. \& Kornfeld, J. M. (1971). Fine structure of resting and germinating Penicilium chrysogenum conidiospores. Protoplasma 73, 443-456.

Mitchell, N. L. \& MCKeen, W. E. (1970). Light and electron microscope studies on the conidium and germ tube of Sphaerotheca macularis. Canadian Journal of Microbiology 16, 273-280.

Morré, D. J., Merritt, W. D. \& Lembi, C. A. (1971). Connections between mitochondria and endoplasmic reticulum in rat liver and onion stem. Protoplasm 73, 43-49.

Ohmori, K. \& GotTlieb, D. (1965). Development of respiratory enzyme activities during spore germination. Phytopathology 55, $1328-\mathrm{I} 336$.

OLIVER, P. T. P. (1972). Conidiophore and spore development in Aspergillus nidulans. Journal of General Microbiology 73, 45-54. 
Remsen, C. C., Hess, W. M. \& Sassen, M. M. A. (1967). Fine structure of germinating Penicillium megasporum conidia. Protoplasma 64, 439-45I.

REYNOLDS, E. S. (1963). The use of lead citrate at high $\mathrm{pH}$ as an electron opaque stain in electron microscopy. Journal of Biophysical and Biochemical Cytology 17, 208-2 12.

Richmond, D. V. \& Pring, R. J. (1971). Fine structure of Botrytis fabae Sardina conidia. Annals of Botany 35, $175-182$.

Sassen, M. M. A., Remsen, C. C. \& Hess, W. M. (1967). Fine structure of Penicillium megasporum conidiospores. Protoplasma 64, 75-88.

Stephens, R. J. \& Bils, R. F. (i965). An atypical mitochondrial form in normal rat liver. Journal of Cell Biology 24, 500-504.

Trinci, A. P. J., Peat, A. \& Banbury, G. H. (1968). Fine structure of phialide and conidiospore development in Aspergillus giganteus Wehmer. Annals of Botany 32, 24I-249.

Weibel, E. R. \& Gomez, D. M. (1962). A principle for counting tissue structures on random sections. Journal of Applied Physiology 17, 343-348.

Weibel, E. R., Kistler, G. S. \& Scherle, W. F. (1966). Practical stereological methods for morphometric cytology. Journal of Cell Biology 30, 23-38.

Weinman-Greenshpan, D. \& Galun, E. (1969). Complementation of nonconidiating mutants of Trichoderma. Journal of Bacteriology 99, 802-806.

WeISBERG, S. H. \& TURIAN, G. (1971). Ultrastructure of Aspergillus nidulans conidia and conidial lomasomes. Protoplasma 72, 55-67.

WeISS, B. (1963). An electron microscope and biochemical study of Neurospora crassa during development. Journal of General Microbiology 39, 85-94. 